\title{
Line Stability Index Based Optimal Placement of Unified Power Flow Controller
}

\author{
Tin Nilar Aung*, Kyaw Myo Lin, Khine Zin Oo \\ Power System Research Unit, Department of Electrical Power Engineering, Mandalay Technological University, Mandalay, Myanmar \\ Email address: \\ nilaraung235@gmail.com (T. N. Aung),kmlep2381@gmail.com (K. M. Lin), khinezinoo.mtu101@gmail.com (K. Z. Oo) \\ ${ }^{*}$ Corresponding author
}

\section{To cite this article:}

Tin Nilar Aung, Kyaw Myo Lin, Khine Zin Oo. Line Stability Index Based Optimal Placement of Unified Power Flow Controller. International Journal of Energy and Power Engineering. Vol. 6, No. 4, 2017, pp. 47-52. doi: 10.11648/j.ijepe.20170604.11

Received: June 21, 2017; Accepted: July 10, 2017; Published: July 31, 2017

\begin{abstract}
Unified power flow controller (UPFC) is a powerful device which can control three power system parameters and it must be located optimally to obtain satisfactory performance due to excessive cost. This paper presents an approach to find the optimal placement of UPFC based on the line stability index. The line stability index can provide accurate information of the stability condition of the lines of the system and can also determine the weakest bus in the system. The index is evaluated for partial network of Myanmar National grid transmission network using MATPOWER Toolbox and a line having largest value of the index is the most critical line where the optimal location of UPFC. The proposed placement approach is shown to be effective for finding appropriate location of UPFC and the effects of UPFC allocation based on line stability index are also demonstrated using Power System Analysis Toolbox (PSAT). The optimal placement of UPFC based on the line stability index resulted in enhancing the power flow control of the system, reducing the system losses as well as improving the system voltage profile.
\end{abstract}

Keywords: Line Stability Index, Optimum Location, Percentage of Series Compensation, PSAT, UPFC

\section{Introduction}

Due to ever increasing load demand, power utilities are now forced to increase the utilization of existing transmission facilities. It is quite difficult to construct new lines due to environmental and economic considerations. As power systems become more complex and heavily loaded, along with economic and environmental constraints, voltage instability become an increasingly serious problem, loading systems to operate close to their limits. Though there are a number of factors directly or indirectly influencing dynamic and static voltage instability, the fundamental reason for this problem is the lack of reactive power reserve [1].

Flexible AC Transmission systems (FACTS) controllers provide fast and reliable control over voltage magnitude, angle and line impedance. FACTS controllers improve the system stability without any change in generation or system parameters [2]. Among the FACTS family, the UPFC is the most powerful device available in terms of its ability to control power system quantities. It is capable of improving reactive power compensation to one node while regulating the active and reactive load flow in the series path of the UPFC [3]. Many models have been developed for the UPFC in load flow studies and have been implemented using a power injection model (PIM) for UPFC in the Newton Raphson load flow algorithm [4].

Moreover, UPFC must be located optimally to obtain satisfactory performance due to excessive cost. From a summarized literature review, the optimal placement of UPFC using differential evolution algorithm to improve the voltage stability is presented in [5]. The hybrid technique based optimal location and sizing of UPFC to improve the dynamic stability is proposed by B. Vijay Kumar [6]. The optimal placement of UPFC using P-V curve and eigenvalue analysis and the critical line using the stability indices are proposed in [7]. Musirin and T. K. Abdul Rahman [8] have discussed a comparison between the fast voltage stability index (FVSI) and line stability index $\left(\mathrm{L}_{\mathrm{mn}}\right)$ with reactive load variation and determines the maximum load bus and also the weak node by using $\mathrm{L}_{\mathrm{mn}}$ index as the FVSI.

In this paper, optimal location of UPFC using line stability index is proposed. The proposed approach has been demonstrated on the partial network of Myanmar National 
grid power system. The rest of the paper is organized as follows: UPFC model is presented in 'Injection Model of UPFC'; the calculation of line stability index and the selection of critical lines in each line of the system are described in 'Line Stability Index Calculation'; the criteria for optimal placement of UPFC using line stability index is proposed in 'Criteria for Optimal Placement of UPFC'; the achievement results and the related discussions are given in 'Results and Discussions' and the paper ends in 'Conclusion'.

\section{Injection Model of UPFC}

The injection model of UPFC proposed by Mete Vural and Mehmet Tu [9] is used in this paper. The model (shown in Figure 1) can be represented in the steady-state condition by two voltage sources representing fundamental components of output voltage waveforms of the two converters and impedances being leakage reactance of the coupling transformers. This model can be used to understand the impact of UPFC on the system in the steady-state conditions. Furthermore, it can easily be incorporated into the steady state power flow model.

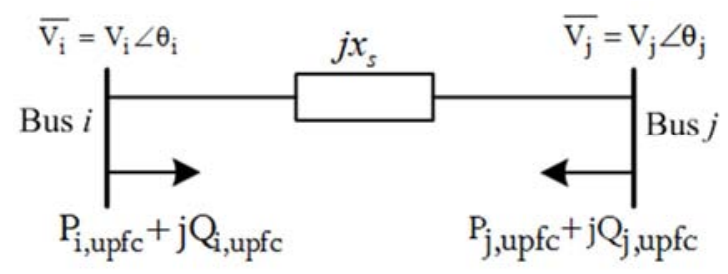

Figure 1. Complete injection model of UPFC.

The active and reactive powers at the connecting buses are:

$$
\begin{gathered}
P_{i, \text { upfc }}=r b_{s e} V_{i} V_{j} \sin \left(\theta_{i j}+\gamma\right) \\
P_{j, \text { upfc }}=-r b_{s e} V_{i} V_{j} \sin \left(\theta_{i j}+\gamma\right) \\
Q_{i, \text { upfc }}=r b_{s e} V_{i}^{2} \cos \gamma \\
Q_{j, \text { upfc }}=-r b_{s e} V_{i} V_{j} \cos \left(\theta_{i j}+\gamma\right)
\end{gathered}
$$

where, $r$ and $\gamma$ are series voltage source coefficient $\left(0 \leqslant r \leqslant r_{\max }\right)$ and series voltage source angle $(0 \leqslant \gamma \leqslant 2 \pi)$, respectively.

If one UPFC is located between node $i$ and node $j$ in a power system, the admittance matrix is modified by adding a reactance equivalent to $X_{s}$, between bus $i$ and bus $j$. The Jacobian matrix is modified by addition of appropriate injection powers. If the linearized load flow model is considered as below:

$$
\left[\begin{array}{c}
\Delta \mathrm{P} \\
\Delta \mathrm{Q}
\end{array}\right]=\left[\begin{array}{cc}
\mathrm{H} & \mathrm{N} \\
\mathrm{J} & \mathrm{L}
\end{array}\right]\left[\begin{array}{c}
\Delta \delta \\
\Delta \mathrm{V}
\end{array}\right]
$$

where, H, N, J, L are the elements of Jacobian matrix.

\section{Line Stability Index Calculation}

This section briefly discusses about line stability index, $\mathrm{L}_{\mathrm{mn}}$ [10] which will be used in selection for optimal placement of UPFC. Most of line stability indices are formulated based on the power transmission concept in a single line. A single line of an interconnected network is illustrated in Figure 2. The line is connected to other lines forming a grid network. Any of the lines from that network can be represented with the following parameters shown in Figure 2.

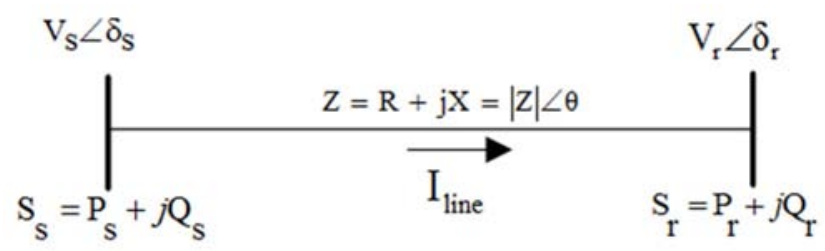

Figure 2. Typical one-line diagram of transmission line.

Utilizing the concept of power flow in the line and analyzing with ' $\pi$ 'model representation, the power flow at the sending and receiving end can be expressed as

$$
\begin{gathered}
\mathrm{S}_{\mathrm{s}}=\frac{\left|\mathrm{V}_{\mathrm{s}}\right|^{2}}{\mathrm{Z}} \angle \theta-\frac{\left|\mathrm{V}_{\mathrm{s}}\right| \mathrm{V}_{\mathrm{r}} \mid}{\mathrm{Z}} \angle\left(\theta+\delta_{\mathrm{s}}-\delta_{\mathrm{r}}\right) \\
\mathrm{S}_{\mathrm{r}}=\frac{\left|\mathrm{V}_{\mathrm{s}}\right|\left|\mathrm{V}_{\mathrm{r}}\right|}{\mathrm{Z}} \angle\left(\theta-\delta_{\mathrm{s}}+\delta_{\mathrm{r}}\right)-\frac{\left|\mathrm{V}_{\mathrm{s}}\right|^{2}}{\mathrm{Z}} \angle \theta
\end{gathered}
$$

From these power equations, it can be separated that real and reactive powers,

$$
\begin{gathered}
\mathrm{P}_{\mathrm{r}}=\frac{\mathrm{V}_{\mathrm{S}} \mathrm{V}_{\mathrm{r}}}{\mathrm{Z}} \cos \left(\theta-\delta_{\mathrm{s}}+\delta_{\mathrm{r}}\right)-\frac{\mathrm{V}_{\mathrm{r}}^{2}}{\mathrm{Z}} \cos \theta \\
\mathrm{Q}_{\mathrm{r}}=\frac{\mathrm{V}_{\mathrm{s}} \mathrm{V}_{\mathrm{r}}}{\mathrm{Z}} \sin \left(\theta-\delta_{\mathrm{s}}+\delta_{\mathrm{r}}\right)-\frac{\mathrm{V}_{\mathrm{r}}^{2}}{\mathrm{Z}} \sin \theta
\end{gathered}
$$

Defining $\delta=\delta_{\mathrm{s}}-\delta_{\mathrm{r}}$ into (9) and solving it for $\mathrm{V}_{\mathrm{r}}$, then,

$$
\mathrm{V}_{\mathrm{r}}=\frac{\mathrm{V}_{\mathrm{S}} \sin (\theta-\delta) \pm \sqrt{\left(\mathrm{V}_{\mathrm{s}} \sin (\theta-\delta)\right)^{2}-4 \mathrm{ZQ} \mathrm{Q}_{\mathrm{r}} \sin \theta}}{2 \sin \theta}
$$

$$
\begin{aligned}
& \text { If } \mathrm{Z}=\sin \theta \mathrm{X}, \\
& \qquad \mathrm{V}_{\mathrm{r}}=\frac{\mathrm{V}_{\mathrm{S}} \sin (\theta-\delta) \pm \sqrt{\left(\mathrm{V}_{\mathrm{s}} \sin (\theta-\delta)\right)^{2}-4 \mathrm{XQ}_{\mathrm{r}}}}{2 \sin \theta}
\end{aligned}
$$

To obtain real values of $\mathrm{V}_{\mathrm{r}}$ in terms of $\mathrm{Q}_{\mathrm{r}}$, the equation must have real roots. Thus, the following conditions, which can be used as a stability criterion, need to be satisfied:

$$
\left(\mathrm{V}_{\mathrm{S}} \sin (\theta-\delta)\right)^{2}-\left(4 \mathrm{XQ} \mathrm{Q}_{\mathrm{r}}\right) \geq 0
$$


(or)

$$
\mathrm{L}_{\mathrm{mn}}=\frac{4 \mathrm{XQ} \mathrm{Q}_{\mathrm{r}}}{\left(\mathrm{V}_{\mathrm{S}} \sin (\theta-\delta)\right)^{2}} \leq 1
$$

The stability criterion is used to find the stability index for each line connected between two bus bars in an interconnected network. As long as the stability index $\mathrm{L}_{\mathrm{mn}}$ remains less than one, the system is stable and when this index exceeds one, the whole system loses its stability and voltage collapse occurs. Thus, the proposed method can be used in this selection for optimal location of UPFC.

A program to calculate the $\mathrm{L}_{\mathrm{mn}}$ index for each line was developed and the following steps are implemented.

(1) Run the load flow program using Newton-Raphson method for the base case.

(2) Evaluate the $L_{m n}$ index in all lines of the system.

(3) Gradually, increase the reactive power in a given bus, keeping the loads on the other nodes constant until power solution stop converge.

(4) Calculate the value of the $L_{m n}$ index for each variation of the load.

(5) Extract the line index that has the highest value; this line is the most critical line for optimal location of UPFC.

(6) Select another load bus and repeat steps 1 to 5 .

\section{Criteria for Optimal Location of UPFC}

UPFC can be theoretically located anywhere along a transmission line but in practically it cannot be incorporated anywhere in the transmission line. In this paper, the following criteria have been considered for optimal location of UPFC on power system transmission lines.

(1) The branches having transformers have not been considered for the UPFC placement.

(2) The line having the highest value of $\mathrm{L}_{\mathrm{mn}}$ is considered the best location for UPFC, followed by other lines having less value.

(3) When two or more cascaded lines in a radial network are considered as the best location, then the line having the highest value of $\mathrm{L}_{\mathrm{mn}}$ of this radial network is considered as the best location for UPFC placement.

(4) The UPFCs are installed one by one and the system improvements are compared with the previous case until there is no improvement considered.

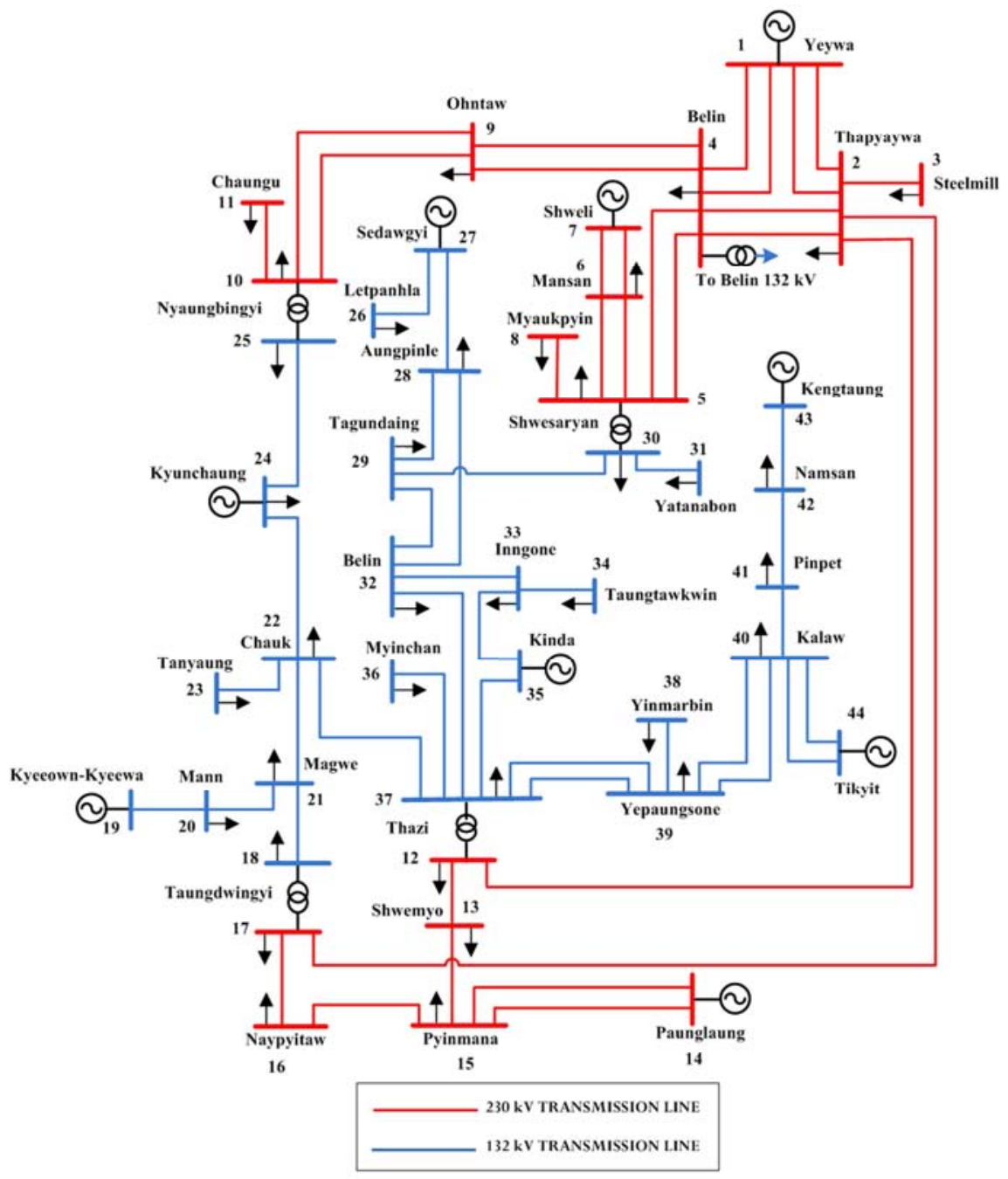

Figure 3. Partial Network of Myanmar National Grid Transmission System. 


\section{Simulation Results and Discussions}

This section demonstrates the implementation of the proposed index on the partial network of Myanmar National grid transmission network for optimal location of UPFCs and the impacts of these devices on the system are also analyzed using PSAT [11].

\subsection{Grid System Scheme}

The one-line diagram of the partial network of Myanmar National grid transmission network is shown in Figure 3, considering with $230 \mathrm{kV}$ and $100 \mathrm{MVA}$ as system base. It consists of 9 generating stations, 52 transmission lines and 5 transformers. Bus-1 (Yeywa) is chosen as slack bus and buses-7, $14,19,24,27,35,43$ and 44 are generator buses and others are load buses. From the results of the load flow study performed on the system without UPFC using PSAT, the total power generated have 933.84 MW and 457.4 Mvar, the total loading level have 914.32 MW and 389.01 Mvar and the total power losses have 19.513 MW and 68.396 Mvar, respectively.

\subsection{Results of Line Stability Index}

To demonstrate the effectiveness of the proposed index, each generated and loaded power is increased gradually until reach the collapse point to verify the unstable lines at high loading conditions. The line stability index, $\mathrm{L}_{\mathrm{mn}}$ is calculated in (12) for all lines in the system using MATPOWER toolbox [12]. The line most suitable for the placement of UPFC has been assigned rank 1; similarly later orders demonstrate the position to be less suitable for the placement of a UPFC. The top 5 rank orders only, have been given in column 2 based on the $\mathrm{L}_{\mathrm{mn}}$ index values which are given in $4^{\text {th }}$ column as shown in Table 1.

Table 1. Rank orders based on line stability index.

\begin{tabular}{llll}
\hline Rank Orders & Line no. & Buses (i-j) & Line Stability Index Value \\
\hline 1 & 29 & $27-28$ & 0.2780609 \\
2 & 27 & $25-24$ & 0.1849889 \\
3 & 38 & $35-33$ & 0.1231328 \\
4 & 36 & $37-32$ & 0.0844889 \\
5 & 39 & $37-35$ & 0.0799052 \\
\hline
\end{tabular}

For the system, the best location for the placement of UPFC is found as line-29 (Sedawgyi-Aungpinle), followed by branches $27,38,36$ and 39 . The numbers of UPFCs installed in the system have been considered by two point of views i.e., the reduction of the total power losses and improvement of the bus voltages of the system. The maximum and the minimum limits of bus voltage magnitude are 1.04 p.u and 0.96 p.u, respectively.

\subsection{System Improvements after Installing the UPFC Devices}

At the initial condition of the system, the total losses of the system are 19.513 MW and 68.396 Mvar, respectively. When one UPFC is placed on the most critical line of the system (line-29), the total losses of the system differ with respect to the original case. And then, the numbers of UPFC devices are increased one by one installation in the system until the 3-UPFCs are placed on the critical lines-29, 27 and 38 of the system as shown in Table 2. The percentage of series compensation of 3-UPFCs is set as $34.21 \%, 72.45 \%$ and $15.25 \%$, respectively. The maximum and the minimum limits of series voltage injected by UPFC are set as 0.3 and -0.3 p.u, respectively.

Table 2. Total power losses of the system with UPFC devices.

\begin{tabular}{lllll}
\hline Losses & Base case & 1-UPFC & 2-UPFCs & 3-UPFCs \\
\hline Active power (MW) & 19.513 & 17.874 & 17.305 & 15.864 \\
Reactive power (Mvar) & 68.396 & 68.011 & 67.964 & 65.466 \\
\hline
\end{tabular}

The results from the above table show that the total power losses of the system are decreased from 19.513 MW and 68.396 Mvar to 15.864 MW and 65.466 Mvar after installation of 3-UPFCs in optimal location of the system.

The all bus voltages are within the allowable limit at the initial condition however three buses 29, 30 and 31 in the network are decreased under the lower limit. When the 3-UPFCs are placed on the most critical lines of the system according to the Table 1, the voltages at the weak buses of the system are significantly improved as shown in Figure 4.

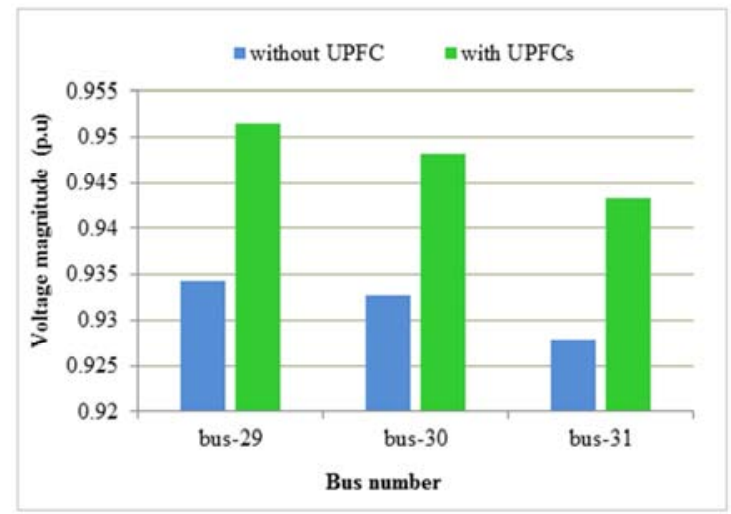

Figure 4. Voltage improvements at weak buses.

The weakest voltage at bus 31 (Yatanarbon) is 0.94326 p.u with $1.7 \%$ increase from the base value. The other weakest voltages at buses 29 and 30 become from 0.93425 p.u and 0.93273 p.u to 0.95145 p.u and 0.94822 p.u, respectively. It can be observed that the entire bus voltages become within the acceptable voltage limit of the system as shown in Figure 5.

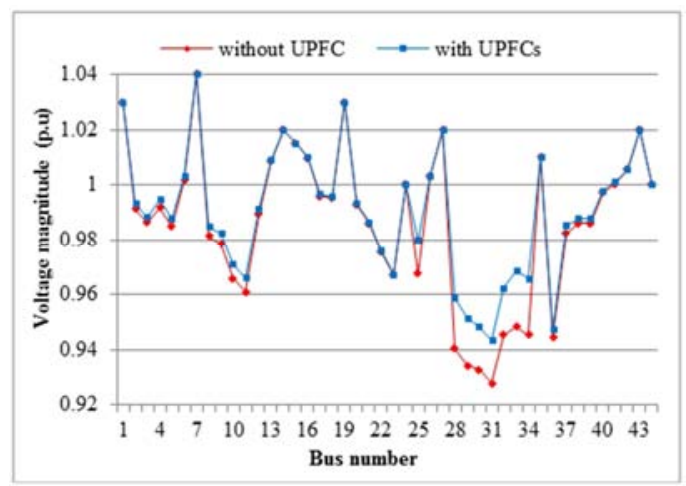

Figure 5. Voltage profile with and without UPFCs. 
Therefore, the installation of 3-UPFCs in optimal location of the system is enough to solve the problem statement of the system by considering the two points of view, the reduction of total power losses and the improvement of voltage profile of the system.

After installation of 3-UPFCs in the optimal location of the system based on the line stability index, the power flow of the system is modified due to the controlled lines flow by the UPFCs. The active and the reactive power flows of the system with and without UPFCs are shown in Figures 6 and 7.

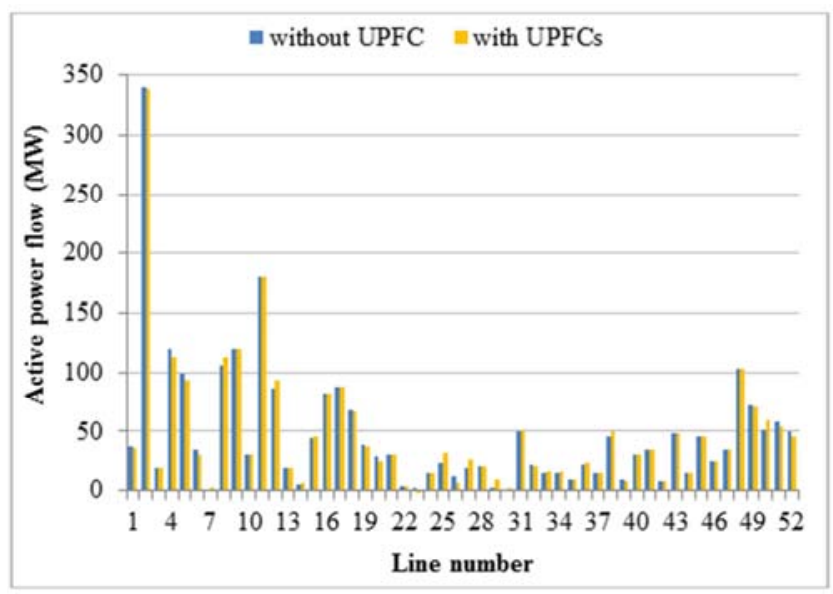

Figure 6. Active power flow with and without UPFCs.

It can be seen from Figures 6 and 7 that line-2 (YeywaBelin) is carrying the highest active and reactive power flows which are 339.97 MW and 140.46 Mvar. The active power flow in line-2 has been reduced to $338.17 \mathrm{MW}$ from the base case.

On the other hand, the reactive power flow in line- 2 has been reduced to 127.75 Mvar. The active and reactive power flows in lines 27, 29 and 38 of the system where the installation of UPFCs has been raised significantly from the initial condition. Therefore, the installation of 3-UPFCs in optimal location of the system provide to control both its active and reactive power flows, high generation of reactive power has been reduced, active power increased and a stable bus voltage magnitude achieved.

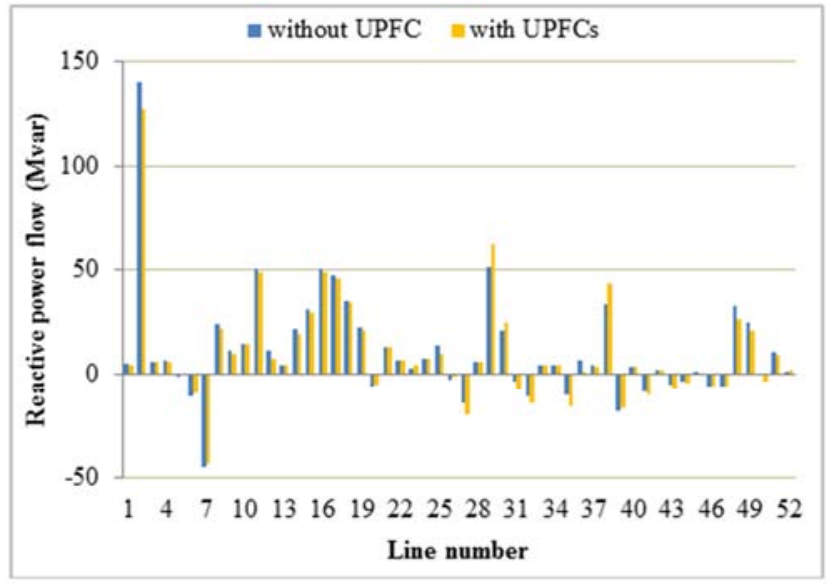

Figure 7. Reactive power flow with and without UPFCs.
The active and reactive power losses are significantly changed after incorporating the UPFC devices in the optimal location of the system as shown in Figures 8 and 9.

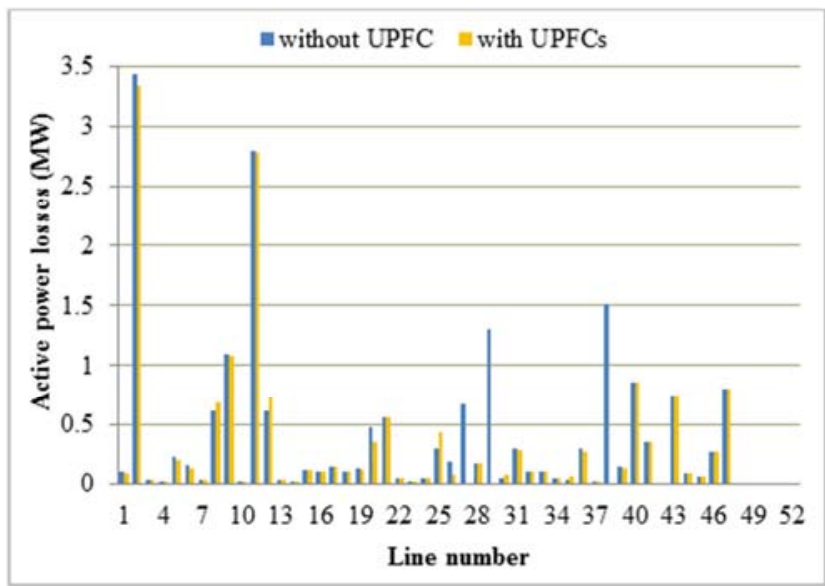

Figure 8. Active power loss with and without UPFCs.

The overall active power losses of the system have been decreased from 19.513 MW to 15.864 MW after incorporating the 3-UPFCs in the optimal location of the system. It can be seen from Figure 8 that the active power losses in lines 27, 29 and 38 of the system have decreased significantly to zero where the installation of optimal placement of UPFCs except the active power losses in lines 8,12 and 25 of the system. The highest active power loss in line- 2 of the system has been decreased from 343.3 MW to 333.2 MW.

From Figure 9, the overall reactive power losses of the system are decreased from 68.396 Mvar to 65.466 Mvar. The highest reactive power loss is 29.124 Mvar which is observed in line 48 at the initial condition. After incorporating the optimal placement of 3-UPFCs in the transmission system, the reactive power loss at line 48 is reduced to 27.42 Mvar.

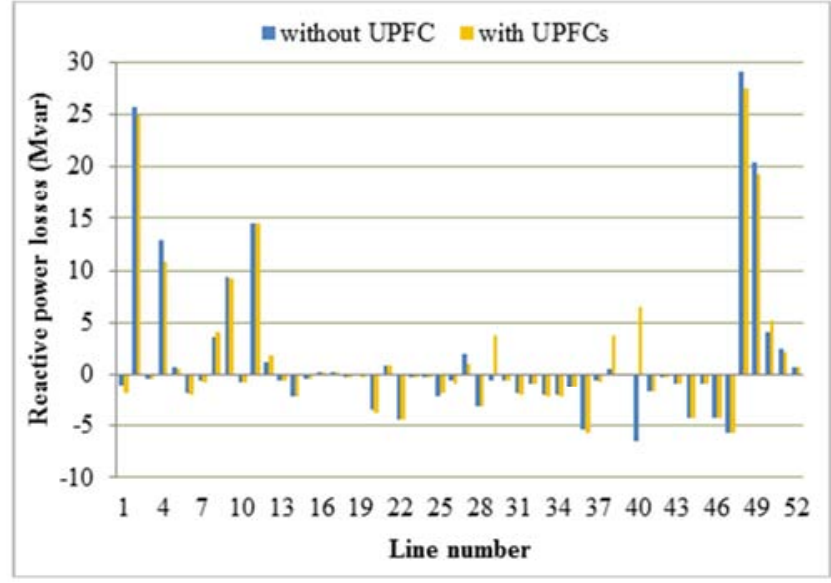

Figure 9. Reactive power loss with and without UPFCs.

Therefore, the optimal location of UPFC based on the line stability index can lead to significant not only the reduction of the transmission losses but also the improvement of the voltage profile and the power flow of the system. 


\section{Conclusion}

In this paper, the proposed approach for successful UPFCs placement based on the line stability index, where the objective is to minimize the line losses and the bus voltage limit violations and the power flow control, is presented. The approach is performed on the partial network of Myanmar National grid transmission system. Results show that the proposed index is capable in determining the suitable location for UPFC devices. Moreover, the optimal location of UPFCs in the system based on the line stability index can regulate the power flow of the system and can increase the voltages at each bus of the system and can reduce the power losses in the system. Therefore, it can be observed that the line stability index approach is effective on the optimal placement of UPFC in the system.

\section{References}

[1] K. R. Padiyar, "FACTS controllers in Power Transmission and Distribution", New Age International Publishers, India, 2007.

[2] Suman Bhowmick, "Flexible AC Transmission Systems (FACTS) Newton Power Flow Modeling of Voltage Source Converter based Controllers", Taylor \& Francis Group, New York, 2016.

[3] L. Gyugyi, T. R. Rietman, A. Edris, C. D. Schauder, D. R Torgerson, and S. L. Williams, "The unified power flow controller: A new approach to power transmission control", IEEE Transactions on Power Delivery, Vol. 10, No. 2, April 1995.

[4] Enrique Acha, Claudio R. Fuerte-Esquivel, Hugo Ambriz-Perez and Cesar Angeles-Camacho, "Flexible AC
Transmission Systems: Modeling and Simulation in Power Networks", John Wiley \& Sons Ltd, England, 2004.

[5] M. Mustapha, B. U. Musa, U. A. Benisheikh and M. A. Sarki, "Optimal placement of UPFC using differential evolution algorithm for improved voltage stability," University of Maiduguri, Faculty of Engineering Seminar Series, vol. 7, July 2016.

[6] B. Vijay Kumar and N. V. Srikanth, "Optimal location and sizing of Unified Power Flow Controller (UPFC) to improve dynamic stability: A hybrid technique", Electrical Power and Energy Systems, pp. 429-438, 2015.

[7] Anju Gupta and P. R. Sharma, "Optimal placement of FACTS devices for voltage stability using line indicators", IEEE fifth Power India Conference, 2012.

[8] I. Musirin and T. K. Abdul Rahamn, "Estimating maximum loadability for weak bus identification using FVSI", IEEE Power Engineering Review, pp. 50-52, November 2002.

[9] Islam Youssef DJILANI KOBIBI, Samir HADJERI, and Mohammed Abdeldjalil DJEHAF, "Study of UPFC Optimal Location Considering Loss Reduction and Improvement of Voltage Stability and Power Flow", Leonardo journal of sciences, issue 24, pp. 85-100, June 2014.

[10] Ebrahim Saadati and Ahmad Mirzaei, "Voltage Stability Indices", International Journal of Science, Engineering and Innovative Research, vol. 8, February 2016.

[11] F. Milano, "PSAT, MATLAB-based Power System Analysis Toolbox," 2009.

(http://www.power.uwaterloo.ca/ fmilano/downloads.html)

[12] Jizhong Zhu, Ray D. Zimmeraman and Carlos E. Murillo-Sanchez, "MATPOWER 5.1 User's Manual", March $20,2015$. 